UVX 2010 (2011) 239-243

DOI: $10.1051 / \mathrm{uvx} / 2011036$

(C) Owned by the authors, published by EDP Sciences, 2011

\title{
Metallic droplets removal induced by pulsed laser: For tokamak application
}

\author{
A. Vatry ${ }^{1,2}$, Ph. Delaporte ${ }^{1}$ and C. Grisolia ${ }^{2}$ \\ ${ }^{1}$ LP3 - UMR 6182 CNRS - Université de la Méditerranée, Case 917, 13009 Marseille, \\ France \\ ${ }^{2}$ CEA, IRFM, 13108 Saint Paul lez Durance, France
}

\begin{abstract}
During tokamak operation, plasma-wall interactions lead to material erosion process and dusts production. For safety reasons the dusts have to be kept in reasonable quantity. The dusts mobilization is a first step to collect them, and the laser is a promising technique for this application. This paper is focused on metallic and metallic oxide pollutants. To optimize the cleaning, physical mechanisms responsible for dust ejection induced by laser have been identified. These removal mechanisms can be very different as a function of the shape and the nature of the particles.
\end{abstract}

\section{INTRODUCTION}

In a tokamak the edge of the plasma is not perfectly confined, thus it can interact with the walls and more particularly with the plasma facing component. These interactions lead to surfaces erosion and dusts production. The particles with sizes from $100 \mathrm{~nm}$ to $100 \mu \mathrm{m}$ can be responsible for safety risk. For safety reasons and to guarantee an optimum reactor functioning, these dusts have to be kept in reasonable quantity [1]. When the surfaces are metallic, the violent loss of plasma energy can induce their melting and micro-sized droplets ejection [2]. Their vaporization can lead to aggregates formation [3]. These metallic particles can be produce in significant quantity and their sizes are in the range of particles which must be collected. In a tokamak, oxidation can occur in case of oxygen ingress, thus metallic oxide.

The laser process seems to be a promising solution to mobilize the dust before their collection. This technique is already used in various fields: nuclear decontamination [4], microelectronics and optics [5]. Then, the laser cleaning has been largely studied and discussed. Further studies are nonetheless necessary to optimize the laser cleaning of metallic droplets and aggregates. This paper presents quantitative measurements of the removal efficiencies. These results are very different as a function of the shape and the nature of the particles, this reveal that the removal mechanisms are different as well. Scanning electron microscope (SEM) observations complete the analysis.

\section{DISPOSITIF EXPERIMENTAL}

All the particle samples were deposited on a silicon substrate $\left(\begin{array}{lll}1 & 0 & 0\end{array}\right)$ from commercial wafers. Three kinds of particles have been studied: tungsten aggregates, tungsten oxide and tungsten micro-sized droplets. The tungsten aggregates are composed by primary particles of few hundreds of nanometers (Fig. 1(a)). The oxide particles are larger, some of them have sizes superior to $3 \mu \mathrm{m}$ (Fig. 1(b)). The tungsten droplets have size from 1 to $8 \mu \mathrm{m}$, and their surface is very smooth, they are covered by thin tungsten nanoaggregates which look like foam (Fig. 1(c)).

This is an Open Access article distributed under the terms of the Creative Commons Attribution-Noncommercial License 3.0, which permits unrestricted use, distribution, and reproduction in any noncommercial medium, provided the original work is properly cited. 
The laser removal experiments were carried out at low repetition rate $(1 \mathrm{~Hz})$ to avoid any cumulative effect. A metallic mask is imaged by lens to obtain a $1 \mathrm{~mm} \times 1 \mathrm{~mm}$ near-uniform irradiation of the substrate. The laser pulse energy was tuned with the aid of a manually operated beam attenuator (Optec, AT4030) to vary the laser fluence. All the removal efficiency experiments were performed in ambient air with five shots. The sample is fixed on a long-range motorized translation stage that allows a precise repositioning of the irradiated zone in front of an optical microscope (Fig. 2). Then, the particles are counted (imaging software), before and after the laser irradiation, and the particle removal efficiency (PRE) is determined. For high-resolution analyses of particles and irradiated material, scanning electron microscopy (SEM-JEOL JSM-6390) was employed.

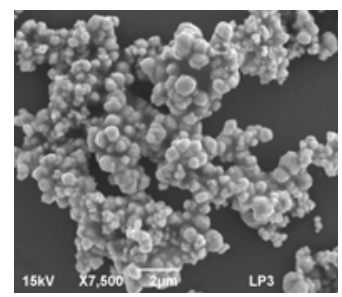

(a)

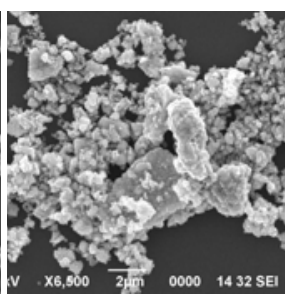

(b)

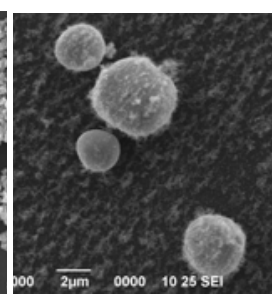

(c)

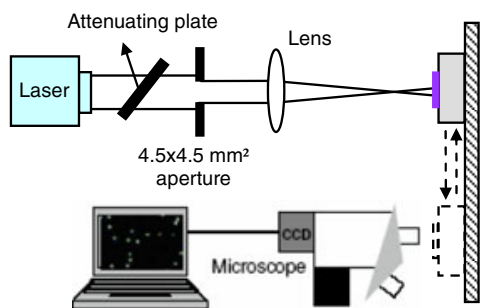

(d)

Figure 1. Particles studied: tungsten aggregates (a), tungsten oxide (b) and tungsten micro-sized droplets (c). Experimental set-up for particle removal efficiency (PRE) measurements (d).

\section{REMOVAL EFFICIENCIES RESULTS}

The particles removal efficiency (PRE) measurements were performed with a XeCl laser (308 nm-50 ns) on the three different kinds of particle, as a function of the fluence. The results are presented in figure 2. We can see that the tungsten oxide particles are very easy to remove. A perfect cleaning is reached for fluences beyond $400 \mathrm{~mJ} / \mathrm{cm}^{2}$. The transition of this curve is quite sharp $\left(\sim 250 \mathrm{~mJ} / \mathrm{cm}^{2}\right)$ for particles not calibrated. These results are not surprising because others studies have already shown the great efficiency of the laser cleaning process in the case of dielectric particles [6]. Satisfying removal efficiencies (80\%) were obtained for the tungsten aggregates for fluences above $800 \mathrm{~mJ} / \mathrm{cm}^{2}$. The slope is low and a perfect removal is never reach for our range of fluences $\left(<1.2 \mathrm{~mJ} / \mathrm{cm}^{2}\right)$. Worst data were measured for tungsten micro-sized droplets where the proportion of removal particles does not exceed $30 \%$.

The three curves have very different shape as a function of the kind of particles. The influence of the particle nature and shape is significant for the laser process efficiency. From these results, we can begin to assume that the physical mechanisms involved in the removal induced by laser are different for these three kinds of particle.

\section{DISCUSSION ON REMOVAL MECHANISMS}

\subsection{Tungsten aggregates}

The scanning electron microscope (SEM) observations allow clearly the identification of the mechanism responsible for the removal of the tungsten aggregates. We can see on the pictures of the figure 3 , that the aggregate is ablated by the irradiations of $450 \mathrm{~mJ} / \mathrm{cm}^{2}$, this fluence corresponds to a removal of $70 \%$. Each irradiation contributes to ablate more and more the particle. Thus, this is obvious that the removal mechanism here is the ablation due to the direct absorption of the beam by the particle. The substrate does not present any damage after the removal. 


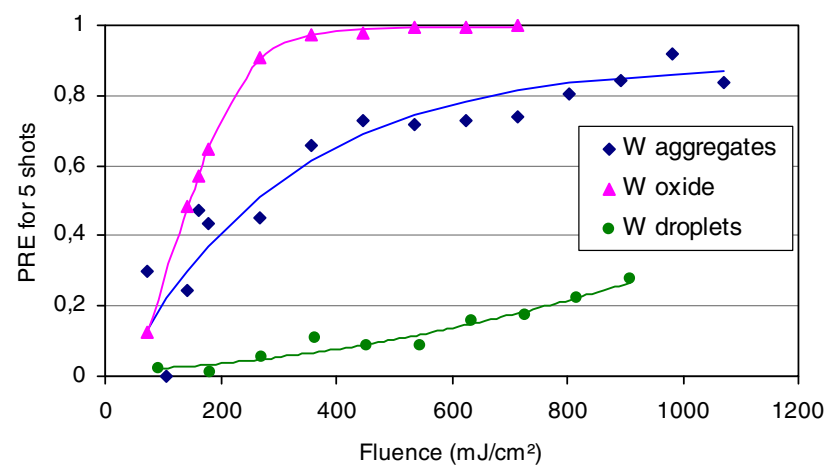

Figure 2. Particle removal efficiencies (PRE) after 5 irradiations performed with a $\mathrm{XeCl}$ laser $(308 \mathrm{~nm}-50 \mathrm{~ns})$, as a function of the fluence, for three kinds of particles: tungsten aggregates, tungsten oxide particles and tungsten micro-sized droplets, deposited on a silicon wafer.
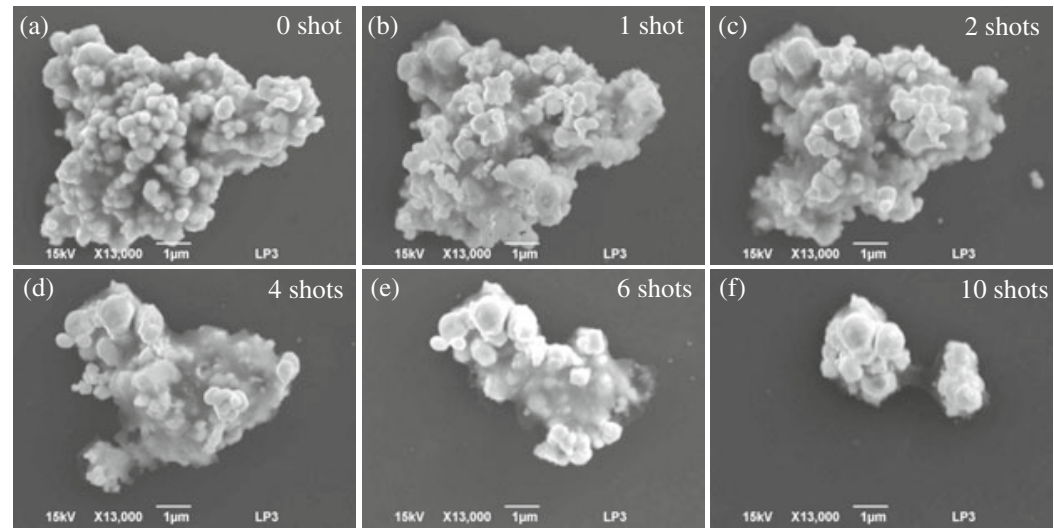

Figure 3. SEM pictures of a tungsten aggregate before irradiation (a) and irradiated by a XeCl laser ( $308 \mathrm{~nm}-50 \mathrm{~ns})$ with a fluence of $450 \mathrm{~mJ} / \mathrm{cm}^{2}$ once (b), twice (c), 4 times (d), 6 times (e) and 10 times (c).

This mechanism explains the removal efficiency results. The slope of the PRE curve is low because small particles can be completely ablated by irradiations whereas larger ones could be only partially ablated. More the fluence is high and larger the particles can be completely ablated. A perfect cleaning is not reach with fluences under $1 \mathrm{~J} / \mathrm{cm}^{2}$ because of the remains of the large particles. We can notice that a higher number of shots could lead to a better efficiency for a same fluence.

\subsection{Tungsten oxide particles}

The oxide particles are dielectric and they do not absorb the laser beam of wavelength $308 \mathrm{~nm}$. Thus the laser ablation of the particle cannot occur, so this removal mechanism is eliminated to explain the removal of these kinds of particle. However, the micro-sized dielectric particles can induce light scattering leading to near-field enhancement. These non linear phenomenons generate an important overintensity underneath the particle and it has been demonstrate that they can lead to explosive evaporation of the humidity trapped at its interface with the substrate [7]. The PRE results are in accordance with this interface removal mechanism because the transition of the curve (10\%-80\%) is sharp for particles not calibrated with such size dispersion (fig. 2). This sudden transition corresponds to the threshold of the explosive vaporization of the humidity by the laser over-intensity. With higher fluences the laser field 
underneath the particle is high enough to induce a local substrate ablation and the particle ejection is due to the momentum transfer from the ablated species. This assumption is confirmed by SEM observation after the irradiations; we can see that the surface of the silicon substrate presents damages which looks like craters. These holes are smaller than the particles because they result from the local ablation.

\subsection{Tungsten droplets}

The behaviour of tungsten droplets under irradiation is very different from the aggregates. The droplets are not ablated by the laser, and no damages are observed on particles collected after the irradiation (Fig. 4(a)). Then it is obvious that the removal mechanism for this kind of particle is not the particle ablation.

Then, we were interested in the substrate under the droplet, to find out if an interface mechanism could be involved in the removal. The SEM observations clearly show that the droplets can be ejected leaving the interface intact. Furthermore the droplet seems to act as a shield protecting the interface in figure 4 (b, c and d), because the thin aggregates under the particle are still on the substrate whereas around it they are left. These observations let to understand that the interface stay cold during the laser irradiation. Thus, all the interface removal mechanisms are eliminated to explain the droplet ejection.

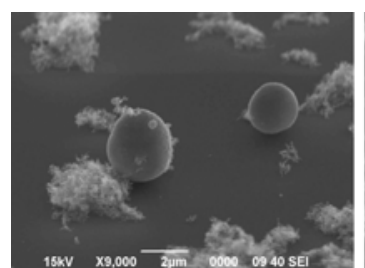

(a)

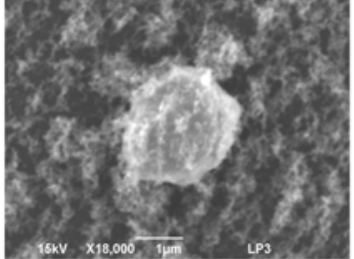

(b)

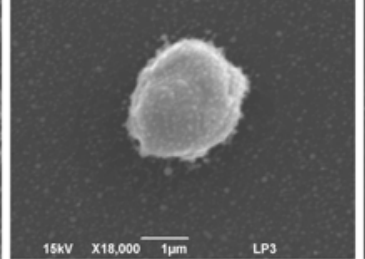

(c)

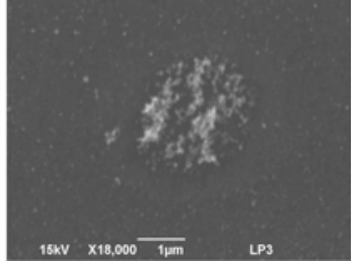

(d)

Figure 4. SEM pictures of tungsten droplets collected intact after their ejection with a substrate set in front the irradiated area (a). A tungsten droplet before irradiation (b) and irradiated by a $\mathrm{XeCl}$ laser ( $308 \mathrm{~nm}-50 \mathrm{~ns})$ with a fluence of $107 \mathrm{~mJ} / \mathrm{cm}^{2}$, twice (c), 4 times (d).

The mechanism already proposed to explain the removal of the metallic droplets is the electrostatic force induced by extraction of photoelectrons [8]. Laser parameters are very influent on the removal efficiency. The photons have to be enough energetic to extract the electrons, and the pulse duration do not exceed more than few nanosecond because the photoelectrons density has to be important. Thus the excimer laser used to performed PRE measurements is not efficient.

\section{SUMMARY AND CONCLUSION}

We have seen that the particles nature, material and shape have a strong influence on the laser-induced removal mechanism. The tungsten aggregates are ablated successively by the laser irradiations, the oxide particles are ejected by interface mechanism, and the tungsten droplets are ejected thanks to the photoelectron extraction and electrostatic force. Understand and identify these removal mechanisms is complex but essential for optimizing the laser process.

\section{Acknowledgments}

This work, supported by Research Federation CNRS-CEA on 'Fusion with Magnetic Confinement - ITER' and the European Communities under the contract of Association between EURATOM and CEA, was carried out within the framework of the European Fusion Development Agreement. The views and opinions expressed herein do not necessarily reflect those of the European Commission. 


\section{References}

[1] J. Roth, E. Tsitrone, A. Loarte et al., J. Nucl. Mater. 1 390-391 (2009) 1-9

[2] A. Herrmann et al., J. Nucl. Mater. 390-391 (2009) 747-750

[3] J.P. Sharpe, D.A. Petti, H.-W. Bartels, Fusion Eng. Des. 63-64 (2002) 153-163

[4] P. Delaporte, M. Gastaud, W. Marine, M. Sentis, O. Uteza, P. Thouvenot, J.L. Alcaraz, J.M. Le Samedy, D. Blin, Appl. Surf. Sci. 298 (2003) 208

[5] W. Zapka, W. Ziemlich, A.C. Tam, Appl. Phys. Lett. 58 (1991) 2217

[6] D. Halfpenny, D. Kane, J. Appl. Phys., 86 (1999) 6641-6646

[7] D. Grojo, Ph. Delaporte, M. Sentis, O.H. Pakarinen, A.S. Foster, Appl. Phys. Lett. 92 (2008) 033108

[8] A. Vatry, A. Marchand, Ph. Delaporte et al., Appl. Surf. Sci. (2010), doi:10.1016/j.apsusc.2010.09.075 